\title{
Innovatives und nachhaltiges Marketing
}

Nachhaltigkeit und Innovation sind in vielen Märkten zu einem Imperativ der Marketingaktivitäten eines Unternehmens geworden. Die Beachtung von sozialen und ökologischen Aspekten ist angesichts sensibilisierter Öffentlichkeit und Verbraucher unabdingbar. Zudem müssen Unternehmen zunehmend innovative und unkonventionelle Wege im Marketing beschreiten, um den Erfolg ihrer Leistungen in den gegenwärtigen Marktumfeldern zu ermöglichen.

In der heutigen Zeit sind Öffentlichkeit und Verbraucher stark für soziale und ökologische Problembereiche sensibilisiert. Die Folgen von Ereignissen wie der Finanzkrise und der Ölkatastrophe im Golf von Mexiko tragen zur weiteren Sensibilisierung bei. Daher sind Unternehmen nicht nur in Branchen, in denen die Relevanz von Nachhaltigkeitsaspekten für Öffentlichkeit und Verbraucher augenscheinlich ist (wie beispielsweise in der Automobilindustrie), zu deren Berücksichtigung angehalten. Vielmehr ist die Beachtung sozialer und ökologischer Aspekte in den meisten Branchen zu einem zentralen Element in der Unternehmenspolitik geworden. Dabei ist es wichtig, dass sich die Nachhaltigkeitsstrategie des Unternehmens auch in den Marketingaktivitäten widerspiegelt. Es obliegt dann insbesondere der Marketingkommunikation durch adäquate und konsistente Maßnahmen die angestrebte Nachhaltigkeitspositionierung in der Wahrnehmung der Öffentlichkeit und der Verbraucher zu erreichen.

Desweiteren gilt es für das Marketing, Herausforderungen wie Marktsättigungstendenzen, Informationsüberlastung und steigende Konsumentenskepsis zu meistern. In diesem Kontext müssen Unternehmen verstärkt innovative Marketinglösungen entwickeln und umsetzen, um die gewünschte Wirkung in den bearbeiteten Märkten zu erzielen. Die rasche Weiterentwicklung von Informations- und Kommunikationstechnologien unterstützt die Entwicklung von Marketinginnovationen, eröffnen sie doch vielfältige Perspektiven und Möglichkeiten für neuartige Marketingideen. Diese können sämtliche Aktionsfelder des Marketing betreffen, die Marketingkommunikation, das Markenmanagement, das interne Marketing etc.

In der vorliegenden Ausgabe der Marketing Review St. Gallen verdeutlichen Beiträge aus Wissenschaft und Praxis die Bedeutung von Nachhaltigkeit und Innovation im Marketing. Sie geben Impulse und Anregungen für die Umsetzung in der Unternehmenspraxis. Hierbei darf das Marketing keinesfalls defensiv vorgehen, vielmehr zeichnet Proaktivität erfolgreiche innovative und nachhaltige Marketingansätze aus.

\section{Prof. Dr. Torsten Tomczak}

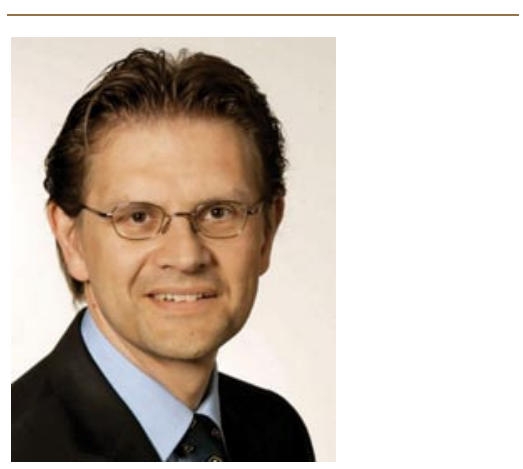

Ordinarius für Betriebswirtschaftslehre mit besonderer Berücksichtigung des Marketing und Direktor der Forschungsstelle für Customer Insight an der Universität St. Gallen

E-Mail: torsten.tomczak@unisg.ch

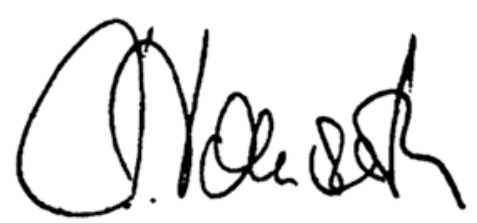

TORSTEN TOMCZAK 